\title{
Epigenetic and Glucocorticoid Receptor-Mediated Regulation of Glutathione Peroxidase 3 in Lung Cancer Cells
}

\author{
Byung Chull An ${ }^{1,6}$, Nak-Kyun Jung ${ }^{1,5,6}$, Chun Young Park ${ }^{2}$, In-Jae Oh ${ }^{3}$, Yoo-Duk Choi ${ }^{2}$, Jae-ll Park ${ }^{4}$, and \\ Seung-won Lee ${ }^{1,5, *}$
}

Glutathione peroxidase 3 (GPx3), an antioxidant enzyme, acts as a modulator of redox signaling, has immunomodulatory function, and catalyzes the detoxification of reactive oxygen species (ROS). GPx3 has been identified as a tumor suppressor in many cancers. Although hyper-methylation of the GPx3 promoter has been shown to down-regulate its expression, other mechanisms by which GPx3 expression is regulated have not been reported. The aim of this study was to further elucidate the mechanisms of GPx3 regulation. GPx3 gene analysis predicted the presence of ten glucocorticoid response elements (GREs) on the GPx3 gene. This result prompted us to investigate whether GPx3 expression is regulated by the glucocorticoid receptor (GR), which is implicated in tumor response to chemotherapy. The corticosteroid dexamethasone (Dex) was used to examine the possible relationship between GR and GPx3 expression. Dex significantly induced GPx3 expression in H1299, H1650, and H1975 cell lines, which exhibit low levels of GPx3 expression under normal conditions. The results of EMSA and ChIP-PCR suggest that GR binds directly to GRE 6 and 7, both of which are located near the GPx3 promoter. Assessment of GPx3 transcription efficiency using a luciferase reporter system showed that blocking formation of the GRGRE complexes reduced luciferase activity by 7-8-fold. Suppression of GR expression by siRNA transfection also induced down-regulation of GPx3. These data indicate that GPx3 expression can be regulated independently via epigenetic or GR-mediated mechanisms in lung cancer cells, and suggest that GPx3 could potentiate glucocorticoid (GC)mediated anti-infla-mmatory signaling in lung cancer cells.

${ }^{1}$ Department of Anatomy, ${ }^{2}$ Department of Pathology, ${ }^{3}$ Department of Internal Medicine, Chonnam National University Medical School, Hwasun 58128, Korea, ${ }^{4}$ Animal Facility of Aging Science, Korea Basic Science Institute, Gwangju 61186, Korea, ${ }^{5}$ Research Institute of Medical Sciences, Chonnam National University, Hwasun 58128, Korea, ${ }^{6}$ These authors contributed equally to this work.

*Correspondence: seunglee@chonnam.ac.kr

Received 29 June, 2016 revised 20 July, 2016; accepted 22 July, 2016; published online 3 August, 2016

Keywords: dexamethasone, gene regulation, glucocorticoids, glucocorticoid receptor, glucocorticoid response element, glutathione peroxidase-3

\section{INTRODUCTION}

Lung cancer is a malignant lung tumor characterized by uncontrolled cell growth in tissues of the lung. If not treated, lung cancer metastasizes beyond the lung into nearby tissue or other parts of the body. This consequence is the leading cause of cancer-related deaths. To improve the survival rate in patients with lung cancer, many studies have sought to identify reliable biological markers of lung cancer (Oh et al., 2014). Recent reports indicate that glutathione peroxidase 3 (GPx3) could be a useful bio-marker for lung cancer, because GPx3 expression is specifically related to lung cancer (Oh et al., 2014). Furthermore, GPx3 has received considerable attention as a tumor suppressor in various cancers during recent years (Barrett et al. 2013; Liu et al., 2015; Qi et al., 2014; Zhang et al., 2010).

GPx3 is the only extracellular secretion-type GPx whose enzymatic function is the detoxification of hydrogen peroxide $\left(\mathrm{H}_{2} \mathrm{O}_{2}\right)$ to protect cells from oxidative damage (Chung et al., 2009). A recent study demonstrated that GPx3 is an effective inhibitor of tumor promotion and progression (Barrett et al., 2013). Moreover, hyper-methylation of the CpG islands in the GPx3 promoter specifically down-regulates its transcription efficiency in many types of mammalian cancer (Chen et al., 2011; He et al., 2011; Mohamed et al., 2014; Peng et al., 2012). In lung cancer cell lines, however, the epigenetic regulation of GPx3 has not been widely described. The present study examined the epigenetic regulation of GPx3 expression using methylation-specific PCR (MSP) and the Illumina Infinium HumanMethylation450 BeadChip array.

Aside from epigenetic regulation, no other mechanisms for the regulation of GPx3 expression have been reported. The core promoter of the GPX3 gene has been determined (Bierl et al., 2004); however, this analysis did not identify the glucocorticoid receptor (GR) as a transcription factor important for the regulation of GPx3 gene expression. However, when we analyzed the GPx3 gene using the Champion ChiP Transcription Factor Search Portal (Qiagen), we identified ten glucocorticoid response elements (GREs): five of GRE sites locate far away from the gene, but the other five locate within the gene (Supplementary Fig. S1). GRE 6 and 7 are of particular interest, as they are located in the downstream regulatory region while the other GREs are located more than $5 \mathrm{~kb}$ (up- or downstream) from the transcription start site (TSS). Glucocorticoids (GCs) can manipulate GR expression and signaling in cells. Moreover, 
GCs are essential for survival and are involved in a variety of physiological processes, including regulation of metabolism, immunity, and apoptosis (McKay and Cidlowski, 1999). Because of their potent pro-apoptotic and anti-inflammatory properties and capability to reduce nausea and acute toxicity in normal tissues, GCs have been widely used as components of chem otherapy regimens for treatment of leukemias, lymphomas, and myelomas (Kim et al., 2004). Although GPx3 was not identified in profiling of primary GR target genes by chromatin immunoprecipitation (ChIP) (Polman et al., 2012; Wang et al., 2004), we decided to further investigate the role of GCs in the regulation of GPx3 expression because the activated GC-GR complex up-regulates the expression of anti-inflammatory proteins in the nucleus (Ingawale et al., 2015), and because GPx3 is a strong tumor suppressor in many types of cancers. Our findings demonstrate that GR can increase GPx3 expression in lung cancer, suggesting that the GPX3 promoter might be a direct target of GC-mediated signaling in tumor cells. Furthermore, these results suggest that GPx3 expression can be regulated by epigenetic or GR-mediated mechanisms in lung cancer cells.

\section{MATERIALS AND METHODS}

\section{Reagents and antibodies}

Human recombinant GR was purchased from Calbiochem (USA). GPx3, GR, and actin antibodies were purchased from Santa Cruz Biotechnology (USA). Anti-mouse antibody conjugated to horseradish peroxidase was obtained from Santa Cruz Biotechnology. For drug treatment, cell lines were treated with $10 \mu \mathrm{M}$ 5-aza-2'-deoxycytidine (5-Aza-CdR) (Sigma, USA) and $100 \mathrm{nM}$ dexamethasone (Dex) (Sigma). Control cells were incubated with DMSO in culture medium.

\section{Cell culture}

H157, H460, A549, H1299, H1650, and H1975 lung cancer cell lines (ATCC, USA) were cultured in appropriate media (RPMI 1640) (Gibco, USA) with $10 \%$ fetal bovine serum (FBS) (Gibco) supplemented with $2 \mathrm{mM} \mathrm{L-glutamine,} 100 \mathrm{U} / \mathrm{ml}$ penicillin, and $100 \mathrm{U} / \mathrm{ml}$ streptomycin (Invitrogen, USA) at $37^{\circ} \mathrm{C}$ in a $5 \% \mathrm{CO}_{2}$ incubator (Hayano et al., 2013).

\section{MSP}

Genomic DNA was prepared from the six lung cancer cell lines using the QIAamp ${ }^{\circledR}$ DNA Mini Kit (Qiagen, USA). Extracted gDNA was bisulfate-modified using the MethylEasy ${ }^{\text {TM }}$ Xceed kit (Human Genetic Signatures, Australia), as described previously (Do et al., 2014; Drini et al., 2011). Briefly, all unmethylated cytosines were deaminated and converted to uracils, whereas 5-methylcytosines remained unaltered. Modified DNA was used as a template for MSP, which was carried out using primers specific for either methylated or modified-unmethylated sequences. Control sample 1, consisting of universal unmethylated DNA (Human Genetic Signatures), was processed by the same procedures as the lung cancer cell gDNAs, whereas control sample 2, consisting of universal methylated DNA (Human Genetic Signatures), was used as a positive control. MSP-specific primers (forward, +18 to +39 /reverse, +209 to +236 ; Table 1 ; Lee et al., 2005) were used to examine whether methylation of the GPx3 promoter CpG region affects GPx3 expression. For PCR, $2 \mu$ l of bisulfite-modified DNA and 20 pmol of each primer were amplified in a total volume of $20 \mu \mathrm{l}$ using AmpONE ${ }^{\text {TM }}$ Taq premix (GeneAll, Korea). PCR conditions were $95^{\circ} \mathrm{C}$ for $10 \mathrm{~min}, 38$ cycles of $95^{\circ} \mathrm{C}$ for $1 \mathrm{~min}$, annealing at $62^{\circ} \mathrm{C}$ (unmethylated) or $66^{\circ} \mathrm{C}$ (methylated) for $1 \mathrm{~min}$ and $72^{\circ} \mathrm{C}$ for $1 \mathrm{~min}$, followed by a final extension step at $72^{\circ} \mathrm{C}$ for $10 \mathrm{~min}$.

\section{DNA methylation arrays}

Samples were bisulfite-converted using the EZ DNA Methylation Kit (Zymo Research, USA) according to the manufacturer's protocol. Controls on the array were used to track the bisulfite conversion efficiency. The Infinium HumanMethylation450 BeadChip array (Illumina Inc., USA) was used to measure genome-wide CpG methylation using beads with target-specific probes designed to interrogate individual $\mathrm{CpG}$ sites on bisulfiteconverted genomic DNA (Bibikova et al., 2011). Measurements were run using DNA samples aliquoted into seven 96-well plates. All plates also contained Illumina controls and two negative controls. Data processing and analysis were performed according to standard protocols (Illumina Inc.). DNA methylation analysis of lung cancer cells ( $\mathrm{H} 460$ and $\mathrm{H} 1299)$ was performed by Macrogen (Korea).

\section{Construction of plasmids}

A GPx3 promoter fragment spanning the $-881 /+1546$ region was amplified by PCR from lung cancer cell genomic DNA using the following primers: -881 (Sacl), 5'-gagctcctattctcaaag gactcctggt-3'; +1546 (Hindlll), 5'-aagcttttccaatgggcttcctgag-3'. The amplified GPx3 promoter fragment was digested with Sacl/HindIII and cloned into the pNL1.1 vector at the Sacl/Hindlll site (Promega, USA) downstream of the nanoluciferase reporter gene. The construct was transformed into Escherichia coli DH5 $\alpha$ cells for amplification. All restriction enzymes were purchased from New England BioLabs (NEB, Ipswich, USA). For PCR, $2 \mu \mathrm{l}$ of cDNA and $20 \mathrm{pmol}$ of each primer were amplified in a total volume of $20 \mu \mathrm{l}$ using Am$\mathrm{pONE}^{\mathrm{TM}}$ Taq premix (GeneAll). PCR conditions were $95^{\circ} \mathrm{C}$ for $10 \mathrm{~min}, 38$ cycles of $95^{\circ} \mathrm{C}$ for $1 \mathrm{~min}$, annealing at $58^{\circ} \mathrm{C}$ for $1 \mathrm{~min}$ and $72^{\circ} \mathrm{C}$ for $1 \mathrm{~min}$, followed by a final extension step at $72^{\circ} \mathrm{C}$ for $10 \mathrm{~min}$ (Voetsch et al., 2007).

\section{Reporter assays}

Lung cancer cells were transfected with 200 ng of nanoluciferase reporter construct (pNL1.1::GPx3 promoter) and 200 ng of firefly luciferase construct encoded by the pGL 4.54 plasmid using Lipofectamine 3000 (Invitrogen). After 2 days of transfection, cells were analyzed using the Nano-Glo Luciferase Assay according to the manufacturer's instructions (Promega) and the Infinite PRO 2000 multimode reader (Tecan, Germany). Measured luciferase values were normalized to the internal firefly luciferase control (Voetsch et al., 2007; Ying et al., 2013).

Reverse transcription polymerase chain reaction (RT-PCR) Total RNA $(1 \mu \mathrm{g})$ from lung cancer cells was reverse transcribed to complementary DNA (cDNA) using Hyperscript ${ }^{\text {TM }}$ RT premix (with oligo dT) (GeneAll) in a final volume of 20 $\mu \mathrm{l}$. This mixture was incubated for $1 \mathrm{~h}$ at $55^{\circ} \mathrm{C}$ and then heated for $10 \mathrm{~min}$ at $95^{\circ} \mathrm{C}$ to inactivate the reverse transcriptase. The resulting cDNAs were used for PCR amplification of the following specific targets: GPx3, GR, and the housekeeping genes glyceraldehyde-3-phosphate dehydrogenase (GAPDH) and actin. Primers were designed using Primer3 so that any genomic DNA product could be distinguished from the target cDNA based on size difference (Table 1). For PCR, $2 \mu \mathrm{l}$ of CDNA and 20 pmol of each primer were amplified in a total volume of $20 \mu \mathrm{l}$ using AmpONE ${ }^{\mathrm{TM}}$ Taq premix (GeneAll). PCR conditions were $95^{\circ} \mathrm{C}$ for $10 \mathrm{~min}, 37$ cycles of $95^{\circ} \mathrm{C}$ for $1 \mathrm{~min}$, annealing at $58^{\circ} \mathrm{C}$ for $1 \mathrm{~min}$ and $72^{\circ} \mathrm{C}$ for 1 $\mathrm{min}$, followed by a final extension step at $72^{\circ} \mathrm{C}$ for $10 \mathrm{~min}$. 
Table 1. Primers of this study

\begin{tabular}{|c|c|c|}
\hline Name & Sequences $\left(5^{\prime}\right.$ to $\left.3^{\prime}\right)$ & \\
\hline GPx3 promoter-F & GAGCTCCTATTCTCAAAGGACTCCTGGT & Cloning \\
\hline GPx3 promoter-R & AAGCTTTTCCAATGGGCTTCCTGAG & \\
\hline GRE6 mutation-F & AAAAATCCACAAGC $\underline{\text { CAGTCTCTGAAATTT }}$ & Mutagenesis \\
\hline GRE6 mutation-R & AAATT TCAGAGACDTG GCTTGTGGAT TTTT & \\
\hline GRE7 mutation-F & TA CC CAGGACCCAC TATIAATCATT A & \\
\hline GRE7 mutation - $\mathrm{R}$ & TAATGATTAIA GTGGGTCCTG GG TA & \\
\hline GRE6 & TCACTACCCAGGACCCACTGTCCTCATTAGCCTG & EMSA \\
\hline GRE7 & AATCCACAAGCTGTTCTCTGAAATTTCCTCC & \\
\hline GRE6 (3'-biotin) & TCACTACCCAGGACCCACTGTCCTCATTAGCCTG- biotin & \\
\hline GRE7 (3'-biotin) & AATCCACAAGCTGTTCTCTGAAATTTCCTCC- biotin & \\
\hline GPx3-F & TGGTCATTCTGGGCTTTCCC & RT-PCR \\
\hline GPx3-R & CCAGAAGAGGCGGTCAGATG & \\
\hline GR-F & TGTTTTGCTCCTGATCTGA & \\
\hline GR-R & TCGGGGAATTCAATACTCA & \\
\hline GAPDH-F & AGGTGAAGGTCGGAGTCA & \\
\hline GAPDH-R & ATACGACTGCAAAGACCC & \\
\hline Actin-F & GGACTTCGAGCAAGAGATGG & \\
\hline Actin-R & AGCACTGTGTTGGCGTCAAG & \\
\hline GPH1010877(+)02A & QIAGEN NOT OPEN & \\
\hline
\end{tabular}

Underlined sequences in the cloning primers indicate restriction enzyme sites, and the underlined sequences in the mutagenesis primers indicate point mutation sequences.

Site-directed mutagenesis of GREs by PCR

Double mutations in GRE6 and GRE7 of the GPx3 promoter were generated by PCR-mediated site-directed mutagenesis. For single mutation of GRE6, the complementary primers contained a triple-base mismatch in GRE6 that converts TGT to CAG using the pNL1.1::GPX3 promoter-GRE (WT) as the template. For double mutations of GRE6 and GRE7, the complementary primers contained a triple-base mismatch in GRE7 that converts GTCC to ATAA using the pNL1.1::GPx3 promoterGRE6 mutant as the template. Briefly, specific PCR was carried out in $20 \mu \mathrm{l}$ mixtures containing $10 \mathrm{ng}$ of plasmid DNA and 20 pmol of each primer using AmpONE ${ }^{\mathrm{TM}}$ Taq premix (GeneAll). PCR conditions were $95^{\circ} \mathrm{C}$ for $10 \mathrm{~min}, 30$ cycles of $95^{\circ} \mathrm{C}$ for 1 min, annealing at $45^{\circ} \mathrm{C}$ for $1 \mathrm{~min}$ and $72^{\circ} \mathrm{C}$ for $5 \mathrm{~min}$, followed by a final extension step at $72^{\circ} \mathrm{C}$ for $10 \mathrm{~min}$. The PCR products of the single and double mutations were collected and purified, treated with Dpnl (Enzynomics, Korea) to remove the original DNA, and then transformed into $\mathrm{DH} 5 \alpha$ cells. The single and double mutations were confirmed by nucleotide sequencing after plasmid isolation (An et al., 2011; 2015).

Gel mobility shift assays

DNA-protein binding was assayed by gel mobility shift EMSA as described previously (Lassar et al., 1991). Binding was carried out using 3'-biotinylated DNA fragments [GRE6 (34 bp) and GRE7 (31 bp)] from the GREs of the GPx3 promoter (GRE6: +1038 to +1054 and GRE7: +1087 to +1105 ) as probes. Typically, binding reactions were run in $20 \mu$ volumes and contained $0.1 \mathrm{ng}$ of 3'-biotinylated DNA fragments (GRE6 and GRE7), $1 \mu \mathrm{g}$ of recombinant GR protein, $1 \mathrm{nmol}$ of Dex diluted in a zinc-containing buffer (10 mM Tris, $\mathrm{pH} 7.6,40 \mathrm{mM} \mathrm{NaCl}, 15$
$\mathrm{mM} \mathrm{ZnCl}_{2}$ ), and $1 \mathrm{mg}$ of poly (dl-dC) under the conditions described previously (Lassar et al., 1991). DNA-protein complexes were resolved on $5 \%$ non-denaturing polyacrylamide gels in $0.25 \times$ TBE $(1 \times$ TBE: $89 \mathrm{mM}$ Tris base, $89 \mathrm{mM}$ boric acid, and 2 $\mathrm{mM}$ EDTA, pH 8.0). DNA-protein complexes were transferred onto a membrane and then fixed by UV crosslinking for $1 \mathrm{mi}-$ nute. The membrane was incubated in blocking buffer and then with streptavidin-HRP monoclonal antibody (Santa Cruz Biotechnology) for $1 \mathrm{~h}$ each. The results were visualized with $\mathrm{Hy}-$ perfilm ECL.

ChIP

ChIP experiments were performed as described previously (Weber et al., 2007). In brief, crosslinked chromatin was sonicated to achieve an average fragment size of $500 \mathrm{bp}$. Starting with $100 \mathrm{mg}$ of chromatin and $5 \mathrm{mg}$ of anti-GR antibody (sc56851; Santa Cruz Biotechnology), $1 \mathrm{ml}$ of ChIP material and 1 $\mathrm{ml}$ of input material were used for reverse transcription polymerase chain reaction (RT-PCR) with specific primers [GPH1010877(+)02A; Qiagen] covering the GPx3 promoter region. Primers specific to GAPDH were used as a negative control, because GAPDH does not possess a GR-binding site.

\section{Western blot analysis}

Following the indicated treatments, cells were washed in phosphate-buffered saline (PBS) and lysed in ice-cold RIPA buffer (20 mM Tris, pH 7.5, $150 \mathrm{mM} \mathrm{NaCl}, 1 \mathrm{mM}$ EDTA, 0.5\% sodium deoxycholate, $1 \%$ IGEPAL CA-630, and $0.1 \%$ sodium dodecyl sulfate) supplemented with protease inhibitor cocktail (Sigma). The cell extracts were cleared by centrifugation at 13,000 rpm for $30 \mathrm{~min}$ at $4^{\circ} \mathrm{C}$, and the protein concentration was quantified 


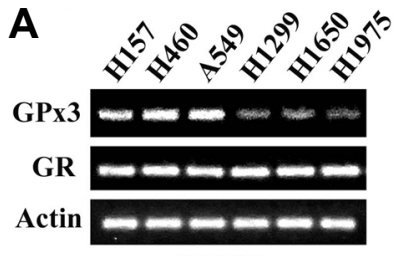

DMSO

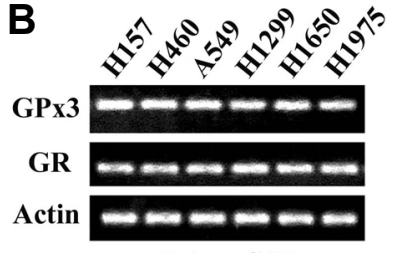

5-Aza-CdR

Fig. 1. Methylation of the GPx3 promoter suppresses its expression in lung cancer cells. GPx3, GR, and actin mRNA expression levels were analyzed by RT-PCR in the H157, H460, A549, H1299, $\mathrm{H} 1650$, and $\mathrm{H} 1975$ lung cancer cell lines. Actin was used as an internal control for normalization. (A) Lung cancer cells treated with DMSO. (B) Lung cancer cells treated with 5-Aza-CdR $(10 \mu \mathrm{M})$ for $24 \mathrm{~h}$.

using a BCA Bio-Rad protein assay kit (Bio-Rad, USA). The cell lysates were resolved on $4 \%$ to $20 \%$ Tris-glycine Ready gels (Bio-Rad) and transferred onto PVDF membranes. Subsequently, the membranes were blocked with $5 \%$ nonfat dry milk in Tris-buffered saline (20 mM Tris base, $\mathrm{pH} 7.5$, and $150 \mathrm{mM}$ $\mathrm{NaCl}$ ) containing $0.05 \%$ Tween 20 (TBS-T) and then probed with primary antibodies (GPx3, GR, and actin) diluted in $1 \%$ nonfat milk in TBS-T. After incubation with primary antibody in TBS-T, the membranes were incubated with the appropriate horseradish peroxidase-conjugated secondary antibodies (Santa Cruz Biotechnology), and the blots were visualized by chemiluminescence (GE Healthcare, USA) (Barrett et al., 2013; Kay et al., 2011).

\section{RESULTS}

GPx3 expression is suppressed by an epigenetic mechanism in lung cancer cells

Suppression of GPx3 expression via an epigenetic mechanism has been reported in many types of human cancer and is proposed to play an important role in tumorigenesis (Lee et al., 2005; Liu et al., 2015; Mohamed et al., 2014; Peng et al., 2012; Yao et al., 2015; Zhang et al., 2010). Therefore, it was of interest to examine whether altered GPx3 expression was a common occurrence in lung cancer cell lines. GPx3 expression was investigated using RT-PCR in six cell lines: H157, H460, A549, $\mathrm{H} 1299, \mathrm{H} 650$, and $\mathrm{H} 1975$. We detected comparably high expression levels of GPx3 mRNA in the H157, H460, and A549 lines, whereas the lines $\mathrm{H} 1299, \mathrm{H} 1650$, and $\mathrm{H} 1975$ exhibited low levels of GPx3 expression (Fig. 1A). GR mRNA was abundantly expressed and expression patterns were similar in all lines (Fig. 1A). Based on these results, the lung cancer cell lines were divided into two groups depending on whether they expressed high or low levels of GPx3 mRNA (Fig. 1A). We hypothesized that the different levels of GPx3 expression might be the result of alternative methylation of the GPx3 promoter (He et al., 2011; Mohamed et al., 2014; Peng et al., 2012). To determine whether methylation was affecting GPx3 expression, we treated the cells with 5-Aza-CdR, a well-known methyltransferase inhibitor. Following treatment, the expression levels of GPx3 significantly increased in the low GPx3 expression level group, while 5-Aza-CdR had little effect on GPx3 expression in the high GPx3 expression level group (Fig. 1B). The expression pattern of GR mRNA was not affected by 5-Aza-CdR treatment (Fig. 1B). This result agreed with the results reported by Kay et al. (2011) demonstrating that some cells, including A549 cells,
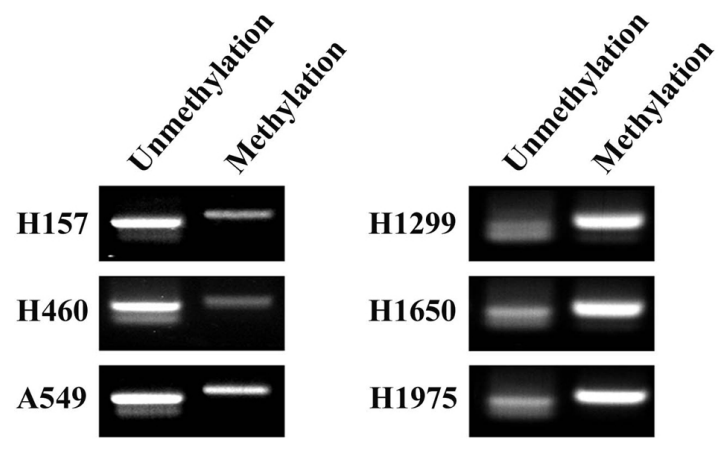

Fig. 2. GPx3 is down-regulated by hyper-methylation of its promoter $\mathrm{CpG}$ in lung cancer cells. Methylation-specific PCR of the GPx3 gene in lung cancer cells. In the gel panels on the left, levels of unmethylated DNA were higher in cells with high levels of GPx3 expression under normal conditions (H157, H460, A549), whereas methylated DNA was only weakly detected. In the panels on the right, cells with low levels of GPx3 expression under normal conditions exhibited low levels of unmethylated DNA and higher levels of methylated DNA.

are insensitive to de-methylation by $5-A z a-C d R$ treatment because these cells showed rare methylation efficiency on CpG islands of the GR promoter. To assess methylation of the GPx3 promoter in these cells, we employed MSP and Infinium HumanMethylation450 BeadChip array. MSP revealed different GPx3 promoter methylation patterns associated with GPx3 expression in each lung cancer cell line. MSP of cells in the low GPx3 expression group revealed hyper-methylation of the GPx3 promoter, whereas cells in the high GPx3 expression group exhibited low levels of methylation (Fig. 2). To identify the methylated sites on the GPx3 promoter, we performed ChIP using the Illumina Infinium HumanMethylation450 BeadChip array, which contains 16 of the methylation sites on the GPx3 gene. We compared the methylation pattern between $\mathrm{H} 460$ and H1299 cell lines which represent a high and a low GPx3 expression group, respectively (Table 2 ). The results showed that six methylation sites located on the CpG island of the GPx3 promoter were hyper-methylated. These results indicate that promoter methylation plays an essential role in the downregulation of GPx3 expression.

GPx3 expression is up-regulated by Dex treatment in lung cancer cells

GCs are one of the steroid hormones that function to maintain homeostasis. The effects of GCs are mediated by ubiquitously expressed GR. The GR is an intracellular ligand-activated transcription factor and member of the nuclear receptor superfamily. The GR is expressed in almost every cell in the body and regulates genes controlling development, metabolism, and the immune response (Taylor et al., 2016). When the GR proteins bind to the GCs, a complex is formed whose primary mechanism of action is the regulation of gene transcription. The activated GC-GR complexes can up- or down-regulate the expression of many genes in the nucleus (Kino et al., 2003; Reddy et al., 2009). However, Dex treatment can repress the expression of mature GR mRNA by $50 \%$ to $80 \%$ in different tissues in humans, mice, and rats (Ramamoorthy and Cidlowski, 2013). In the present study, the putative GREs of the GPx3 gene were mapped using the Champion ChiP Transcription Factor Search 

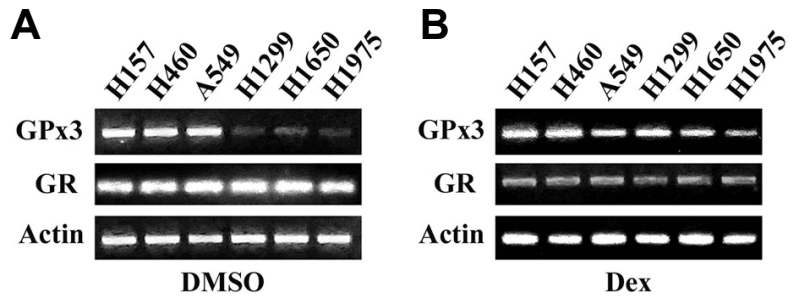

C

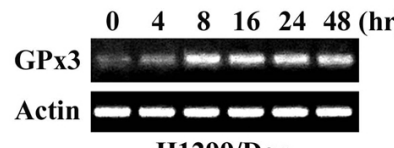

H1299/Dex $\begin{array}{llllll}0 & 4 & 8 & 16 & 24 & 48\end{array}(\mathrm{hr})$

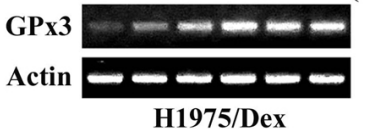

Fig. 3. GPx3 is up-regulated by Dex treatment in lung cancer cells. GPx3, GR, and actin mRNA expression levels were analyzed by RT-PCR in the H157, H460, A549, H1299, H1650, and H1975 lung cancer cell lines. Actin was used as an internal control for normalization. (A) Lung cancer cells treated with DMSO. (B) Lung cancer cells treated with Dex $(100 \mathrm{nM})$ for $24 \mathrm{~h}$. (C) To examine the time course of the effect of Dex, GPx3 expression was assessed in $\mathrm{H} 1299$ and $\mathrm{H} 1975$ at the indicated time points after treatment.

Portal (Qiagen), which identified ten GREs spread across the GPx3 gene (Supplementary Fig. S1). To determine the role of GRs in mediating GPx3 expression, Dex was used to examine the possible relationship between GR and GPx3 expression. Dex treatment of the lung cancer cell lines showed that GPx3 expression levels significantly increased in the low GPx3 expression level group, while the high GPx3 expression level group was not affected and GR expression was slightly decreased (Fig. 3B). In agreement with this finding, a reduction in GR mRNA expression by Dex treatment in lung cancer was reported previously (Ramamoorthy and Cidlowski, 2013). Time course analysis showed that Dex strongly induced GPx3 expression within 8-16 h in lung cancer cells (Fig. 3C).

Recombinant hGR protein binds specifically to GREs of the GPx3 promoter

Binding of recombinant hGR protein to GREs in the GPx3 promoter was studied by EMSA using 3'-biotinylated GRE6 (34 bp: sequence +2194 to +2329 ) or $3^{\prime}$-biotinylated GRE7 (31 bp: +2233 to +2298 ) DNA probes. As shown in Supplementary Fig. S2, both the GRE6 and GRE7 DNA probes shifted significantly in the presence of Dex due to the formation of a complex between the GREs and hGR protein. The ChIP-PCR revealed that the GR protein bound specifically to the GREs of the GPx3 promoter region in lung cancer cells (Fig. 4). To verify the specificity of the GR-GRE complex on the GPx3 promoter region, we employed primer pairs [Qiagen; GPH1010877(+)02A; Table 1] specific for the GPx3 promoter region (1 kb). A primer pair for GAPDH was used as a negative control, as this gene does not contain a GRE. In addition, a GPx3 exon-specific primer pair was used as a second negative control. Together, these results indicate that GR binds specifically to GREs in the GPx3 promoter in lung cancer cells.

Enhanced transcription of GPx3 is associated with GR binding

The functional importance of the GREs was then tested by mutational analysis. Mutations targeted to GR-binding motifs on

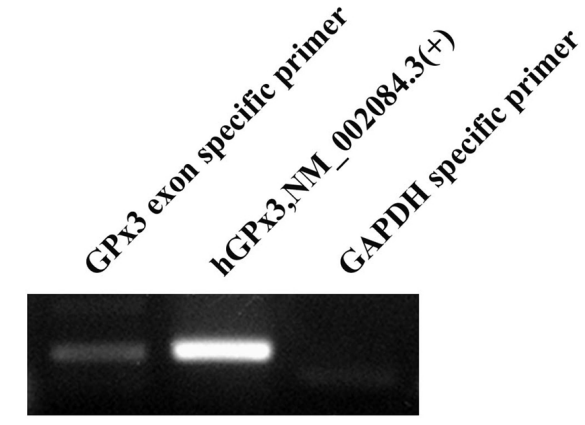

Fig. 4. hGR protein binds specifically to GREs on the GPx3 promoter in lung cancer cells. ChIP was performed using A549 cells. ChIP-PCR was then performed using a primer pair [GPH1010877(+)02A] specific for GRE6 and GRE7 in intron 1 of the GPx3 gene. As negative controls, primers for GAPDH, which does not possess a GRE, and an exon-specific GPx3 primer pair were used.

both GRE6 and GRE7 are described in Table 2. The promoter activities of the wild-type GRE (WT) and double-mutated GRE (MT) constructs were tested in lung cancer cells. Interestingly, Dex-induced transcription of the GPx3 gene was accompanied by decreased transcription of GR mRNA, as assessed by RTPCR (Fig. 3B). The GR-mediated increase in GPx3 expression shown in Fig. 3 was then confirmed by a cell-based luciferase reporter assay, which showed that the double mutation of GRE6 and GRE7 led to significantly reduced GR-mediated transactivation of the GPx3 promoter (Fig. 5). The GRE (WT) construct exhibited approximately 7-8-fold higher luciferase activity than the GRE (MT) construct (Fig. 5). Together, the EMSA, ChIP, and luciferase reporter assay results suggest that the GR binds specifically to GREs in the GPx3 promoter, leading to induction of GPx3 expression.

Silencing of GR reduces GPx3 expression in lung cancer cells

To confirm this conclusion, we also performed transient siRNA transfection to specifically suppress GR expression. In both groups of lung cancer cells, especially the A549 and in H1975 lines, transfection of siRNA against GR resulted in decreased GR expression and decreased transcription of the GPx3 gene as assessed by RT-PCR and Western blot (Fig. 6).

\section{DISCUSSION}

GPx3 is the only extracellular glutathione peroxidase to play a critical role in detoxifying ROS and protecting DNA from damage caused by ROS in mammalian cells (Cheng et al., 1992). ROS can induce DNA mutations that are most frequently observed in proto-oncogenes and tumor suppressor genes (Hussain and Harris, 1998). The increased production of ROS accompanying inflammation may also contribute to malignancy (Barrett et al., 2013). Recent studies suggest that GPx3 acts as a potent tumor suppressor (Barrett et al., 2013; Qi et al., 2014; Wang et al., 2012; Yu et al., 2007). Barrett et al. (2013) demonstrated that GPx3 acts as a tumor suppressor during inflammatory carcinogenesis in cancer cells via detoxification of ROS, thus preventing DNA damage that leads to tumor initiation.

Many studies have reported silencing of GPx3 caused by hyper-methylation of the promoter in human prostate cancer, 
GR Potentiates Expression of GPx3 Acts As a Tumor Suppressor

Byung Chull An et al.

Table 2. Methylation analysis of the GPx3 CpG island using the Illumina Infinium HumanMethylation450 BeadChip array

\begin{tabular}{llll}
\hline Probe ID & $\mathrm{H} 1299-\mathrm{H} 460$ (delta_mean) & $\mathrm{H} 1299-\mathrm{H} 460(>0.2)$ & Map Information \\
\hline cg04201367 & -0.012540408 & hyper & 151028823 \\
cg08891071 & 0.53373774 & hyper & 151020348 \\
*g10802379 & 0.411667333 & & 151020834 \\
cg11109721 & 0.002466637 & & 151028344 \\
cg12684668 & 0.014101828 & hyper & 151023905 \\
cg13081720 & 0.7993864 & & 151020329 \\
$\operatorname{cg} 16627309$ & -0.013691176 & hyper & 151019782 \\
cg17261508 & 0.345409464 & hyper & 151019973 \\
cg17820459 & 0.49086509 & hyper & 151020970 \\
cg18849169 & 0.261546521 & hyper & 151020220 \\
cg21504918 & 0.456547762 & hyper & 151020440 \\
* & 0.561578642 & & 151020767 \\
cg21516478 & -0.043050576 & & 151019861 \\
cg22005145 & 0.042117262 & hyper & 151019582 \\
*g26638444 & 0.505308151 & hyper & 151020619 \\
cg26891370 & 0.287892398 & 151021111 \\
\hline
\end{tabular}

* The lllumina $450 \mathrm{~K}$ probes in the $\mathrm{CpG}$ island of the GPx3 promoter are designated by an asterisk.
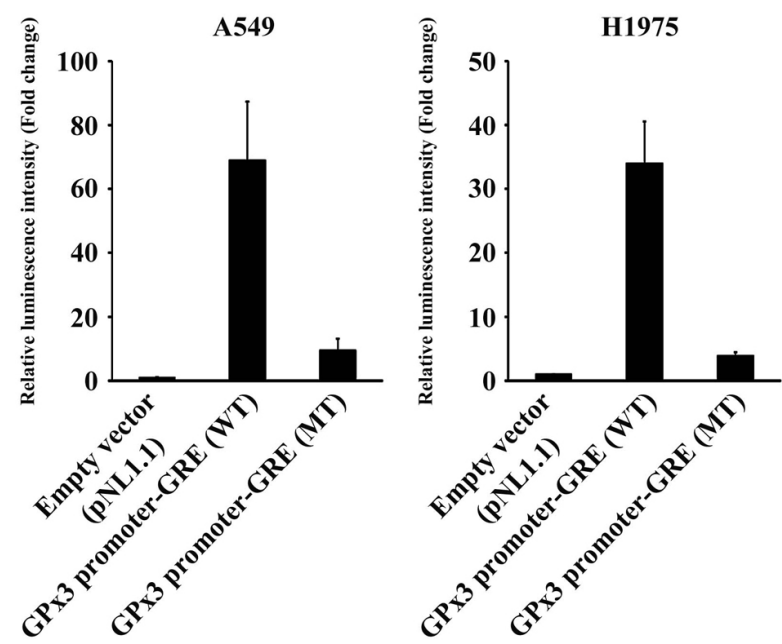

Fig. 5. Functional analysis of GR and GREs in lung cancer cells. The effect of dual mutation of GRE6 and GRE7 on the GPx3 promoter was determined using a luciferase reporter system in lung cancer cells. A reporter plasmid with nano luciferase [pNL1.1::GPx3 promoter-GRE (WT) or (MT)] and a control plasmid with firefly luciferase ( $p G L 4.54$ ) were co-transfected into lung cancer cells. Nano luminescence was determined and normalized to the firefly luciferase signal. The fold-inductions were then calculated by normalization to the pNL1.1 control.

breast cancer, lung cancer, ovarian clear cell adenocarcinoma, gastric carcinoma, and Barrett's disease (Jee et al., 2009; Lee et al., 2005; Lodygin et al., 2005; Saga et al., 2008; Yu et al., 2007; Zhang et al., 2010). Methylation of the GPx3 promoter significantly reduced its transcription activity, and the methyla-
A

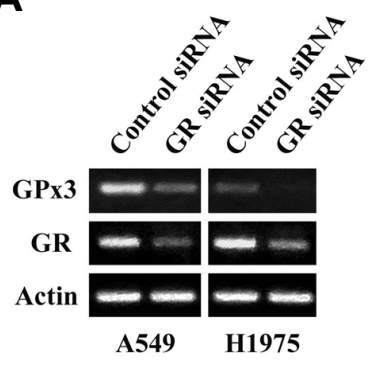

B

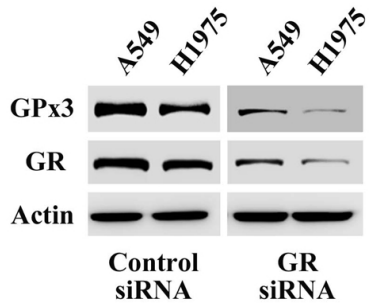

Fig. 6. Suppression of GR mRNA reduces GPx3 expression levels in lung cancer cells. Effect of GR knockdown on GPx3 expression in lung cancer cells. (A) A549 and H1975 cells were transfected with siRNA against GR or a control siRNA, and expression of GPx3 was evaluated $48 \mathrm{~h}$ later by RT-PCR. As shown, the levels of GPx3 were significantly decreased in the presence of GR siRNA, but not in the presence of control siRNA. (B) Western blot analysis of total cell lysates of A549 and H1975 cells transfected with GR siRNA. As shown, transfection with GR siRNA decreased the levels of GPx3 expression in lung cancer cells.

tion frequency and the methylated sites on the promoter showed wide diversity depending on the type of cancer ( $\mathrm{He}$ et al., 2011; Mohamed et al., 2014; Peng et al., 2012). In the current study, we verified the epigenetic regulation of GPx3 in lung cancer cells with low levels of GPx3 expression by showing that the GPx3 promoters in these cells exhibited high methylation frequency.

Despite the growing interest in GPx3 as a molecule functionally involved in tumor suppression, information about GPx3 gene regulation other than the epigenetic control remains limited. Here, we demonstrated for the first time that GR-mediated 
up-regulation of GPx3 is independent of the epigenetic regulation of GPx3 in lung cancer cells.

Leggas et al. (2009) demonstrated that Dex pre-treatment decreases hematopoietic toxicity and improves the efficacy of the chemotherapeutic regimen in patients with metastatic nonsmall cell lung cancer. In addition, GCs have been widely used as components of chemotherapeutic regimens for the treatment of leukemias, lymphomas, and myelomas due to their potent pro-apoptotic and anti-inflammatory properties and ability to reduce nausea and acute toxicity in normal tissues (Tothova et al., 2011). Dex treatment also suppresses non-small cell lung cancer proliferation and cell cycle progression through inhibition of the ERK/MAPK pathway (Greenberg et al., 2002). Our results showed that the specific binding of GR protein and GREs (GRE6 and GRE7) was verified, and that GPx3 expression is significantly induced by GC-GR complex activity. In addition, it is suggested that GR-mediated up-regulation of GPx3 is independent of the epigenetic regulation of GPx3.

In conclusion, our demonstration that GR mediates upregulation of the GPx3 gene in lung cancer cells provides important insights into cancer biology. GPx3 is well known to act as an antioxidant and tumor suppressor in cancer cells (Barrett et al., 2013; Chung et al., 2009; Liu et al., 2015; Qi et al., 2014; Zhang et al., 2010). Considering this, we postulate that the effects of GCs on cancer response to chemotherapy are accomplished at least partially via GR-mediated expression of GPX3. This finding could provide useful information for developing anti-cancer therapies designed to enhance GPx3 expression by GC.

Note: Supplementary information is available on the Molecules and Cells website (www.molcells.org).

\section{ACKNOWLEDGMENTS}

This study was supported by a grant (CRI 15023-21) from the Chonnam National University Hospital Biomedical Research Institute to SWL and by a fellowship from the Research Institute of Medical Sciences, Chonnam National University to NKJ. This work was also supported by the Basic Science Research Program through the National Research Foundation of Korea (NRF) funded by the Ministry of Education, Science and Technology (grant number: 2013R1A1A2007187) to IJO.

\section{REFERENCES}

An, B.C., Lee, S.S., Lee, J.T., Hong, S.H., Wi, S.G., and Chung, B.Y. (2011). Engineering of 2-Cys peroxiredoxin for enhanced stresstolerance. Mol. Cells 32, 257-264.

An, B.C., Lee, S.S., Jung, H.S., Kim, J.Y., Lee, Y., Lee, K.W., Lee, S.Y., Tripathi, B.N., and Chung, B.Y. (2015). An additional cysteine in a typical 2-Cys peroxiredoxin of Pseudomonas promotes functional switching between peroxidase and molecular chaperone. FEBS Lett. 589, 2831-2840.

Barrett, C.W., Ning, W., Chen, X., Smith, J.J., Washington, M.K., Hill, K.E., Coburn, L.A., Peek, R.M., Chaturvedi, R., Wilson, K.T., et al. (2013). Tumor suppressor function of the plasma glutathione peroxidase gpx3 in colitis-associated carcinoma. Cancer Res. 73, 1245-1255.

Bibikova, M., Barnes, B., Tsan, C., Ho, V., Klotzle, B., Le, J.M., Delano, D., Zhang, L., Schroth, G.P., Gunderson, K.L., et al. (2011). High density DNA methylation array with single CpG site resolution. Genomics 98, 288-295.

Bierl, C., Voetsch, B., Jin, R.C., Handy, D.E., and Loscalzo, J. (2004). Determinants of human plasma glutathione peroxidase (GPx-3) expression. J. Biol. Chem. 279, 26839-26845.

Chen, B., Rao, X., House, M.G., Nephew, K.P., Cullen, K.J., and Guo, Z. (2011). GPx3 promoter hypermethylation is a frequent event in human cancer and is associated with tumorigenesis and chemotherapy response. Cancer Lett. 309, 37-45.

Cheng, K.C., Cahill, D.S., Kasai, H., Nishimura, S., and Loeb, L.A (1992). 8-Hydroxyguanine, an abundant form of oxidative DNA damage, causes G-T and A-C substitutions. J. Biol. Chem. 267, 166-172.

Chung, S.S., Kim, M., Youn, B.S., Lee, N.S., Park, J.W., Lee, I.K., Lee, Y.S., Kim, J.B., Cho, Y.M., Lee, H.K., et al. (2009). Glutathione peroxidase 3 mediates the antioxidant effect of peroxisome proliferator-activated receptor gamma in human skeletal muscle cells. Mol. Cell. Biol. 29, 20-30.

Do, H., Wong, N.C., Murone, C., John, T., Solomon, B., Mitchell, L., and Dobrovic, A. (2014). A critical re-assessment of DNA repair gene promoter methylation in non-small cell lung carcinoma. Sci. Rep. 4, 4186

Drini, M., Wong, N.C., Scott, H.S., Craig, J.M., Dobrovic, A., Hewitt, C.A., Dow, C., Young, J.P., Jenkins, M.A., Saffery, R., et al. (2011). Investigating the potential role of genetic and epigenetic variation of DNA methyltransferase genes in hyperplastic polyposis syndrome. PLoS One 6, e16831.

Greenberg, A.K., Hu, J., Basu, S., Hay, J., Reibman, J., Yie, T.A., Tchou-Wong, K.M., Rom, W.N., and Lee, T.C. (2002). Glucocorticoids inhibit lung cancer cell growth through both the extracellular signal-related kinase pathway and cell cycle regulators. Am. J. Respir. Cell Mol. Biol. 27, 320-328.

Hayano, T., Garg, M., Yin, D., Sudo, M., Kawamata, N., Shi, S., Chien, W., Ding, L.W., Leong, G., Mori, S., et al. (2013). SOX7 is down-regulated in lung cancer. J. Exp. Clin. Cancer Res. 32, 17

He, Y., Wang, Y., Li, P., Zhu, S., Wang, J., and Zhang, S. (2011). Identification of GPX3 epigenetically silenced by $\mathrm{CpG}$ methylation in human esophageal squamous cell carcinoma. Dig. Dis. Sci. 56, 681-688

Hussain, S.P., and Harris, C.C. (1998). Molecular epidemiology of human cancer: contribution of mutation spectra studies of tumor suppressor genes. Cancer Res. 58, 4023-4037.

Ingawale, D.K., Mandlik, S.K., and Patel, S.S. (2015). An emphasis on molecular mechanisms of anti-inflammatory effects and glucocorticoid resistance. J. Complement. Integr. Med. 12, 1-13.

Jee, C.D., Kim, M.A., Jung, E.J., Kim, J., and Kim, W.H. (2009). Identification of genes epigenetically silenced by CpG methylation in human gastric carcinoma. Eur. J. Cancer 45, 1282-1293.

Kay, P., Schlossmacher, G., Matthews, L., Sommer, P., Singh, D., White, A., and Ray, D. (2011). Loss of glucocorticoid receptor expression by DNA methylation prevents glucocorticoid induced apoptosis in human small cell lung cancer cells. PLoS One 6, e24839.

Kim, Y.S., Park, J.S., Jee, Y.K., and Lee, K.Y. (2004). Dexamethasone inhibits TRAIL- and anti-cancer drugs-induced cell death in A549 cells through inducing NF-kappaB-independent cIAP2 expression. Cancer Res. Treat. 36, 330-337.

Kino, T., Souvatzoglou, E., De Martino, M.U., Tsopanomihalu, M., Wan, Y., and Chrousos, G.P. (2003). Protein 14-3-3s interacts with and favors cytoplasmic subcellular localization of the glucocorticoid receptor, acting as a negative regulator of the glucocorticoid signaling pathway. J. Biol. Chem. 278, 25651-25656.

Lassar, A.B., Davis, R.L., Wright, W.E., Kadesch, T., Murre, C., Voronova, A., Baltimore, D., and Weintraub, H. (1991). Functional activity of myogenic HLH proteins requires hetero-oligomeri- zation with E12/E47-like proteins in vivo. Cell 66, 305-315.

Lee, O.J., Schneider-Stock, R., McChesney, P.A., Kuester, D. Roessner, A., Vieth, M., Moskaluk, C.A., and El-Rifai, W. (2005). Hypermethylation and loss of expression of glutathione peroxidase-3 in Barrett's tumorigenesis. Neoplasia 7, 854-861.

Leggas, M., Kuo, K.L., Robert, F., Cloud, G., deShazo, M., Zhang, R., Li, M., Wang, H., Davidson, S., and Rinehart, J. (2009). Intensive anti-inflammatory therapy with dexamethasone in patients with non-small cell lung cancer: effect on chemotherapy toxicity and efficacy. Cancer Chemother. Pharmacol. 63, 731-743.

Liu, Q., Jin, J., Ying, J., Sun, M., Cui, Y., Zhang, L., Xu, B., Fan, Y., and Zhang, Q. (2015). Frequent epigenetic suppression of tumor suppressor gene glutathione peroxidase 3 by promoter hypermethylation and its clinical implication in clear cell renal cell carcinoma. Int. J. Mol. Sci. 16, 10636-10649.

Lodygin, D., Epanchintsev, A., Menssen, A., Diebold, J., and Hermeking, $H$. (2005). Functional epigenomics identifies genes frequently silenced in prostate cancer. Cancer Res. 65, 4218-4227.

McKay, L.I., and Cidlowski, J.A. (1999). Molecular control of im- 
mune/inflammatory responses: interactions between nuclear factor- $\mathrm{kB}$ and steroid/receptor-signaling pathways. Endocr. Rev. 20, 435-459.

Mohamed, M.M., Sabet, S., Peng, D.F., Nouh, M.A., El-Shinawi, M., and El-Rifai, W. (2014). Promoter hypermethylation and suppression of glutathione peroxidase 3 are associated with inflammatory breast carcinogenesis. Oxid. Med. Cell Longev. 2014, 787195.

Oh, I.-J., Kim, H.-E., Song, S.-Y., Na, K.-J., Kim, K.-S., Kim, Y.-C., and Lee, S.-W. (2014). Diagnostic value of serum glutathione peroxidase 3 levels in patients with lung cancer. Thoracic Cancer 5 , 425-430.

Peng, D.F., Hu, T.L., Schneider, B.G., Chen, Z., Xu, Z.K., and ElRifai, W. (2012). Silencing of glutathione peroxidase 3 through DNA hypermethylation is associated with lymph node metastasis in gastric carcinomas. PLoS One 7, e46214.

Polman, J.A., Welten, J.E., Bosch, D.S., de Jonge, R.T., Balog, J., van der Maarel, S.M., de Kloet, E.R., and Datson, N.A. (2012). A genome-wide signature of glucocorticoid receptor binding in neuronal PC12 cells. BMC Neurosci. 13, 118.

Qi, X., Ng, K.T.P., Lian, Q.Z., Liu, X.B., Li, C.X., Geng, W., Ling, C.C., Ma, Y.Y., Yeung, W.H., Tu, W.W., et al. (2014). Clinical significance and therapeutic value of glutathione peroxidase 3 (GPx3) in hepatocellular carcinoma. Oncotarget 5, 11103-11120.

Ramamoorthy, S., and Cidlowski, J.A. (2013). Ligand-induced repression of the glucocorticoid receptor gene is mediated by an NCoR1 repression complex formed by long-range chromatin interactions with intragenic glucocorticoid response elements. Mol. Cell. Biol. 33, 1711-1722.

Reddy, T.E., Pauli, F., Sprouse, R.O., Neff, N.F., Newberry, K.M., Garabedian, M.J., and Myers, R.M. (2009). Genomic determination of the glucocorticoid response reveals unexpected mechanisms of gene regulation. Genome Res. 19, 2163-2171.

Saga, Y., Ohwada, M., Suzuki, M., Konno, R., Kigawa, J., Ueno, S., and Mano, H. (2008). Glutathione peroxidase 3 is a candidate mechanism of anticancer drug resistance of ovarian clear cell adenocarcinoma. Oncol. Rep. 20, 1299-1303.

Taylor, K.M., Ray, D.W., and Sommer, P. (2016). Glucocorticoid receptors in lung cancer: new perspectives. J. Endocrinol. 229, R17-28.
Tothova, V., Isola, J., Parkkila, S., Kopacek, J., Pastorek, J., Pastorekova, S., and Gibadulinova, A. (2011). Glucocorticoid receptor-mediated transcriptional activation of S100P gene coding for cancer-related calcium-binding protein. J. Cell. Biochem. 112 3373-3384.

Voetsch, B., Jin, R.C., Bierl, C., Benke, K.S., Kenet, G., Simioni, P., Ottaviano, F., Damasceno, B.P., Annichino-Bizacchi, J.M., Handy, D.E., and Loscalzo, J. (2007). Promoter polymorphisms in the plasma glutathione peroxidase (GPx-3) gene: a novel risk factor for arterial ischemic stroke among young adults and children. Stroke 38, 41-49.

Wang, J.C., Derynck, M.K., Nonaka, D.F., Khodabakhsh, D.B., Haqq, C., and Yamamoto, K.R. (2004). Chromatin immunoprecipitation (ChIP) scanning identifies primary glucocorticoid receptor target genes. Proc. Natl. Acad. Sci. U SA 101, 15603-15608.

Wang, H., Luo, K., Tan, L.Z., Ren, B.G., Gu, L.Q., Michalopoulos, G., Luo, J.H., and Yu, Y.P. (2012). p53-induced gene 3 mediates cell death induced by glutathione peroxidase 3 . J. Biol. Chem. 287, 16890-16902.

Weber, M., Hellmann, I., Stadler, M.B., Ramos, L., Pääbo, S. Rebhan, M., and Schübeler, D. (2007). Distribution, silencing potential and evolutionary impact of promoter DNA methylation in the human genome. Nat. Genet. 7, 457-466.

Yao, D.M., Zhou, J.D., Zhang, Y.Y., Yang, L., Wen, X.M., Yang, J., Guo, H., Chen, Q., Lin, J., and Qian, J. (2015). GPX3 promoter is methylated in chronic myeloid leukemia. Int. J. Clin. Exp. Pathol. 8, 6450-6457.

Ying, C.Y., Dominguez-Sola, D., Fabi, M., Lorenz, I.C., Hussein, S. Bansal, M., Califano, A., Pasqualucci, L., Basso, K., and DallaFavera, R. (2013). MEF2B mutations lead to deregulated expression of the oncogene BCL6 in diffuse large B cell lymphoma. Nat Immunol. 14, 1084-1092.

Yu, Y.P., Yu, G., Tseng, G., Cieply, K., Nelson, J., Defrances, M., Zarnegar, R., Michalopoulos, G., and Luo, J.H. (2007). Glutathione peroxidase 3 , deleted or methylated in prostate cancer, suppresses prostate cancer growth and metastasis. Cancer Res. 67 8043-8050.

Zhang, X., Yang, J.J., Kim, Y.S., Kim, K.Y., Ahn, W.S., and Yang, S. (2010). An 8-gene signature, including methylated and downregulated glutathione peroxidase 3 , of gastric cancer. Int. J. Oncol. $36,405-414$ 